\title{
An Efficient Mobility Management Scheme for Two-Level HMIPv6 Networks*
}

\author{
Xuezeng Pan, Zheng Wan, Lingdi Ping, and Fanjun Su \\ College of Computer Science, Zhejiang University, Hangzhou, P.R. China \\ zhengwan66@yahoo.com.cn, \{xzpan, ldping\}@zju.edu.cn, \\ sufanjun@163.com
}

\begin{abstract}
In this paper, we propose an efficient mobility management scheme in a two-level Hierarchical Mobile IPv6 (HMIPv6) architecture. A mobile node chooses a suitable Mobility Anchor Point (MAP) according to its average dwell time and its number of connecting correspondent nodes. Furthermore, a higher MAP may adjust the threshold of MAP selection algorithm periodically according to its traffic load.
\end{abstract}

\section{Introduction}

Hierarchical Mobile IPv6 (HMIPv6) [1] was proposed to reduce the amount of registration between the mobile node $(\mathrm{MN})$, its home agent and correspondent nodes (CNs). To improve the performance of HMIPv6 further, many schemes [2-5] proposed that the MN choose a suitable MAP according to its mobility characteristics. For example, Kawano et al. [4-5] introduced a multilevel HMIPv6 architecture and a speed based MAP selection algorithm. However, these algorithms did not consider the influence of traffic parameter of an $\mathrm{MN}$, i.e. the number of connecting CNs. Thus reduction of registration cost that achieved by mobility based algorithm is limited.

In this paper, we propose a mobility and traffic based MAP selection algorithm for two-level HMIPv6 architecture. In addition, a dynamic adjustment mechanism is introduced to reduce the registration cost further, in which a higher MAP may adjust the threshold of MAP selection periodically according to its traffic load.

\section{Proposed Scheme}

\subsection{MAP Selection}

We combine the number of CNs $\left(N_{c}\right)$ and dwell time $\left(T_{f}\right)$ as the parameter for MAP selection (called "combined measure", referred to as " $C$ "). The following equation is used to compute $C$.

$$
C=\left(1+N_{c}\right) / T_{f}
$$

This work was funded in part by Huawei Funds for Science and Technology (YJCB2004025SP) and Science and Technology Plan of Zhejiang Province (2005C21002). 
The MAP selection strategy is defined as:

$$
M A=\left\{\begin{array}{cc}
M A P_{h} & C>T_{c} \\
M A P_{l} & C \leq T_{c}
\end{array}\right.
$$

where $M A$ indicates the chosen MAP and $T_{c}$ denotes the threshold of combined measure. $M A P_{h}$ and $M A P_{l}$ denote higher MAP and lower MAP, respectively.

\subsection{Dynamic Adjustment}

If $N_{c}$ of each $\mathrm{MN}$ often changes, a fixed threshold can not minimize the inter-domain registration cost. Based on this observation we propose the dynamic adjustment mechanism. Each higher MAP periodically checks the number of its serving MNs $\left(N_{m}\right)$ and compares $N_{m}$ with its capacity $\left(C_{m a p}\right)$. Assume the checking interval is $I$. We use "Sat" to denote the saturation degree of a higher MAP, which is equal to $N_{m} / C_{m a p}$. The higher MAP adjusts $T_{c}$ according to its current $S a t$ as follows:

$$
T_{c_{-} n}=\left\{\begin{array}{lc}
T_{c_{-} o} \times(1+\beta) & \text { Sat }>\text { Sat }_{h} \\
T_{c_{-} o} & \text { Sat } \\
T_{c_{-} o} \times(1-\beta) & \text { Sat } \geq \text { Sat }_{l}<\text { Sat }_{l}
\end{array}\right.
$$

where $T_{c_{\_} n}$ and $T_{c_{-} o}$ are the new threshold and the old threshold. $S a t_{h}$ and $S_{a t} t_{l}$ denote two thresholds for adjustment decision. $\beta$ indicates the degree of adjustment and is a positive constant less than 1 .

To deploy proposed scheme, two fields are included into the MAP option:

- $\quad L$ : a bit lies after "R" bit, indicating the level of an MAP. "1" refers to a higher MAP and " 0 " refers to a lower MAP.

- Threshold: updated $T_{c}$ for MAP selection. It is valid when "L" bit is set. It is 64 bits long and lies before "lifetime" field.

\section{Performance Evaluation}

\subsection{Simulation Model}

There are 16 higher MAPs distributed in the simulated grid network. Each higher MAP is connected by 16 lower MAPs and each lower MAP covers 4 subnets. There are "high speed MNs" and "low speed MNs", with dwell time $T_{f 1}$ and $T_{f 2}$ respectively, which follow the uniform distribution with parameters [5s,10s] and $[10 \mathrm{~s}, 15 \mathrm{~s}]$. We set the ratio of high speed MNs to low speed MNs to 1:1. Mobile nodes are also divided into two classes according to their traffic parameters. $N_{c}$ for low traffic MNs may be 0 and 1 , and $N_{c}$ for high traffic MNs may be 5 and 6 . The ratio of these two traffic classes is defined as a simulation parameter $\left(R_{T}\right)$.

We consider three schemes. The first is the traditional mobility based MAP selection scheme, in which high/low speed MNs choose higher/lower MAPs. The second is the combined measure based MAP selection algorithm. And the last scheme 
deploys dynamic adjustment mechanism on the basis of the second scheme. The three schemes are referred to as Speed scheme, Comb scheme and Comb_D scheme. The simulation time is 5,000 seconds. Each experiment is repeated ten times and the average result is presented. Table 1 summarizes the major parameters.

Table 1. Simulation parameters

\begin{tabular}{l|l|l}
\hline Parameter(s) & Description & Value \\
\hline$N_{M}$ & The number of simulated MNs & 200 to 1000 \\
$R_{T}$ & Ratio for low to high traffic classes & $5: 1$ to $1: 5$ \\
$\beta$ & Adjustment degree for dynamic mechanism & 0.2 \\
$I$ & Interval for a higher MAP to check $N_{m}$ & 10 \\
$T_{c}$ & Initial threshold for MAP selection & 0.4 \\
a t $_{h}$ & Up threshold for dynamic mechanism & 1.0 \\
at $_{l}$ & Down threshold for dynamic mechanism & 0.8 \\
$C_{\text {map }}$ & Capacity of higher MAP & $\mathrm{N}_{\mathrm{M}} / 16$ \\
\hline
\end{tabular}

\subsection{Simulation Results}

From Fig. 1 we find that the number of binding updates (referred to as $N_{b}$ ) of the three solutions all increases with increasing $N_{M}$. The percentage reductions of registration cost obtained by Comb and Comb_D schemes over Speed scheme are approximately $25 \%$ and $45 \%$ respectively.

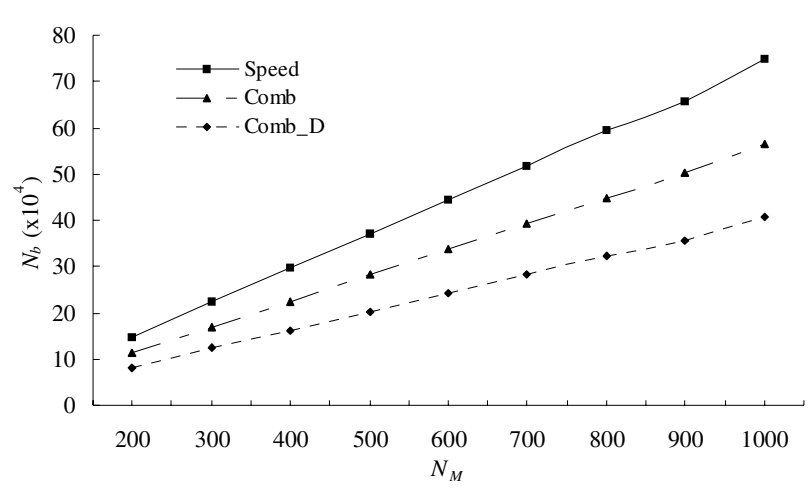

Fig. 1. $N_{b}$ when $N_{M}$ varies $\left(R_{T}=1: 1\right)$

We modify $R_{T}$ to simulate the scenario in which $N_{c}$ of the MN changes. Served by the same MAP, an MN with more $\mathrm{CNs}$ issues more binding updates. Thus more high traffic MNs indicates larger registration cost in Speed scheme, as shown in Fig. 2. On the contrary, the Comb scheme shows its adaptability to various ratios of traffic classes because high traffic MNs try to choose higher MAPs according to our novel MAP selection algorithm. As for Comb_D scheme, when the number of high traffic MNs is low, the higher MAP will reduce its threshold to allow more MNs to 
be served. As the number of high traffic MNs increases, more MNs are originally served by higher MAPs. Thus performance gain by deploying dynamic adjustment decreases.

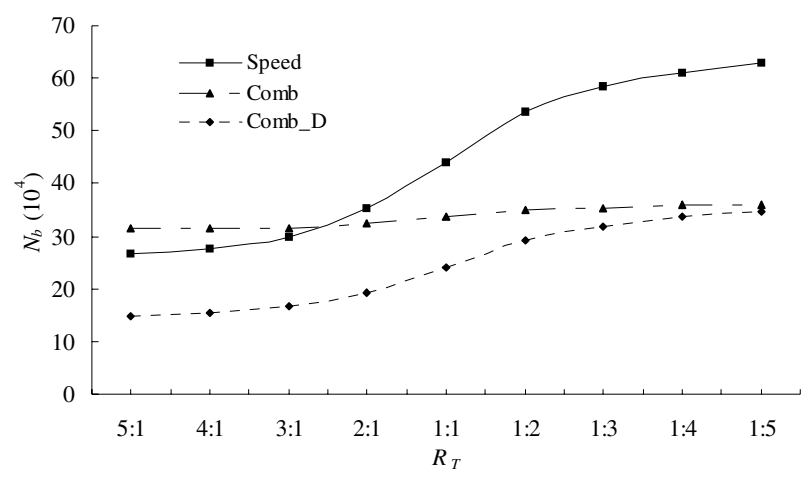

Fig. 2. $N_{b}$ when $R_{T}$ varies $\left(N_{M}=600\right)$

\section{Conclusion}

In this paper, we propose a mobility and traffic based MAP selection algorithm and a dynamic adjustment mechanism to reduce the registration cost outside MAP domains in a two-level HMIPv6 architecture. Taking traffic parameter into account, the novel MAP selection algorithm achieves better performance in reducing overall registration cost than mobility based MAP selection algorithm. In addition, dynamic adjustment prevents saturation degree of the higher MAP from being a small value so that it reduces the registration cost further.

\section{References}

1. H. Soliman, C. Castelluccia, K. El-Malki, and L. Bellier, "Hierarchical Mobile IPv6 mobility management," IETF RFC 4140, Aug. 2005.

2. S.H. Hwang, B.K. Lee, Y.H. Han, and C.S. Hwang, "An adaptive hierarchical mobile IPv6 with route optimization," IEEE Vehicular Technology Conference (VTC), Apr. 2003.

3. T. Kumagai, T. Asaka, and T. Takahashi, "Location management using mobile history for hierarchical mobile IPv6 networks,” IEEE GLOBECOM, pp.1585-1589, Nov. 2004.

4. K. Kawano, K. Kinoshita, and K. Murakami, "A multilevel hierarchical distributed IP mobility management scheme for wide area networks," International Conference on Computer Communications and Networks (ICCCN), pp.480-484, Oct. 2002.

5. K. Kawano, K. Kinoshita, and K. Murakami, "A mobility-based terminal management in IPv6 networks," IEICE Transactions on Communications, vol. E85-B, no.10, pp.2090 2099, Oct. 2002. 